\title{
Les cas de succès en transport urbain durable : une liste exhaustive des types de facteurs
}

\author{
Jean Mercier et Mario Carrier \\ Université Laval
}

\section{INTRODUCTION}

\section{Même si l'augmentation de la production de GES en transport urbain augmente au niveau de la planète dans son ensemble, nous avons plusieurs exemples de villes ou de régions métropolitaines qui semblent, mieux que d'autres, contrôler cette augmentation.}

Deux éléments se conjuguent pour faire du transport urbain durable un objectif incontournable des politiques publiques urbaines d'aujourd'hui. D'abord, de plus en plus des habitants de la planète vivent dans des villes, et on serait déjà arrivé à environ la moitié d'urbains par rapport à la population mondiale totale. Le deuxième élément a trait à la production de gaz à effet de serre (GES), puisque c'est dans le domaine du transport que le contrôle de leur augmentation est le plus difficile, si on le compare aux deux autres grands secteurs, soit la production d'énergie et les processus industriels. Donc, ce qui va se passer dans les villes, du point de vue du transport, va être un élément crucial des objectifs de contrôle des GES et de leur éventuelle réduction.

Même si l'augmentation de la production de GES en transport urbain augmente au niveau de la planète dans son ensemble, nous avons plusieurs exemples de villes ou de régions métropolitaines qui semblent, mieux que d'autres, contrôler cette augmentation. Nous avons un moyen fiable pour mesurer le succès des villes à cet égard, soit les données qui nous viennent des statistiques de ce que nous appelons les «parts modales». Les parts modales nous informent sur les différents moyens de transport qui sont utilisés dans un territoire urbain pour assurer la mobilité de ses habitants. Par exemple, une ville qui connaît du succès en transport urbain durable utilisera moins l'automobile, reconnue comme le moyen de transport le moins durable, et utilisera davantage des moyens de transport reconnus comme durables, comme le transport en commun, le vélo ou la marche. Une ville qui est reconnue comme ayant du succès en matière de transport urbain durable, Singapour, montre une part modale en transport en commun d'environ $50 \%$, alors qu'une ville ayant peu de succès en la matière, comme Phoenix en Arizona, n'en a que $5 \%$. Nous sommes privilégiés, dans le domaine du transport urbain, d'avoir ces statistiques assez objectives aux fins de comparaisons (même si des écarts peuvent exister pour ce qui est de la mesure des parts modales, comme la délimitation du territoire étudié, qui peut varier d'une ville à l'autre).

À travers des études accumulées au cours des dernières années, notre groupe de recherche sur les instruments de politiques publiques pour un transport urbain durable, financé par le Conseil de recherches en sciences humaines du Canada, a développé un modèle qui se veut exhaustif sur les éléments qui expliquent le succès des villes en matière de transport urbain durable. En effet, même si notre intérêt initial portait sur les instruments de politiques publiques (comme la réglementation, les incitations économiques ou la planification du territoire, par exemple) pour expliquer le succès de certaines villes en matière de transport urbain durable, nous nous sommes rendu compte qu'il y avait une série d'autres facteurs qui expliquaient, aussi, le succès des villes en la matière. Le présent article vise à faire l'inventaire exhaustif de ces types de facteurs. Nous avons donc divisé ces facteurs en cinq 
catégories (ou types) de facteurs, qui sont les suivants : 1) les contraintes naturelles; 2) les contraintes créées par l'intervention humaine; 3) les dynamiques institutionnelles; 4) Le choix des politiques publiques; 5) le choix des instruments de politiques publiques. La Figure 1 illustre le continuum qui structure cette liste de facteurs, soit un continuum qui part d'éléments qui sont relativement peu modifiables, relevant alors d'un déterminisme, et qui vont vers des éléments qui sont davantage malléables, c'est-à-dire qui peuvent être soumis à un volontarisme. Ainsi, il est pratiquement impossible d'agir sur les contraintes naturelles d'une ville, comme son insularité par exemple, alors qu'il apparaît plus facile d'agir au niveau des instruments d'intervention, comme la réglementation ou les interventions économiques pour encourager ou décourager tel ou tel comportement en transport (interdire l'entrée de l'automobile dans certains secteurs de la ville ou, encore, en accroître le coût, par exemple).

Nous présentons donc maintenant ces cinq catégories de facteurs, en commençant donc par les moins malléables. Nous prétendons que tous les types de facteurs sont couverts, même si certains des sous-ensembles de nos catégories peuvent être absents dans le présent texte. Autrement dit, nous pensons qu'il ne manque aucun type de facteur important dans notre liste pour expliquer le succès (ou l'insuccès) des villes en transport urbain durable.

Figure 1 - Pyramide des contraintes et opportunités

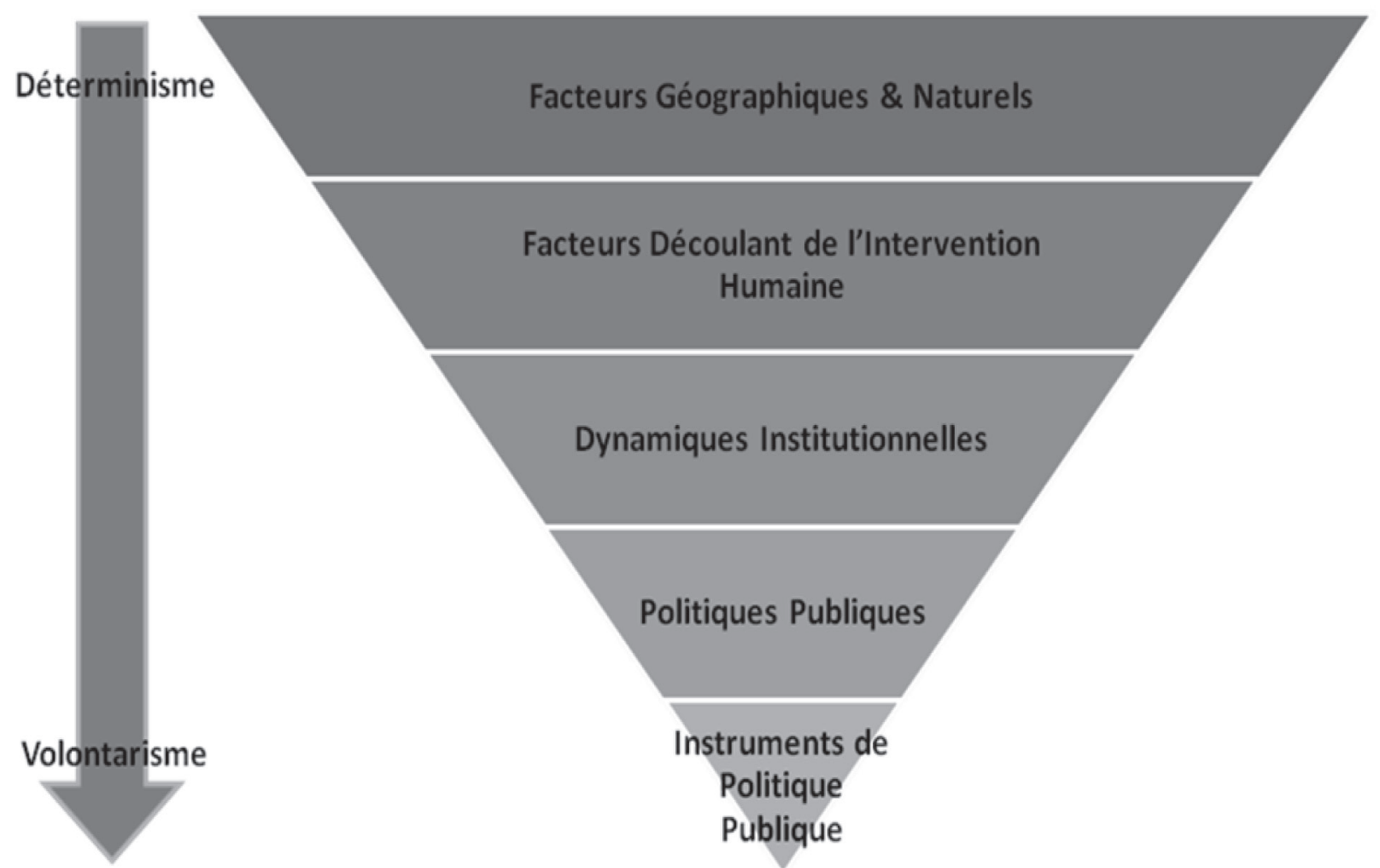




\section{LES CONTRAINTES NATURELLES}

Nous entendons par «contraintes naturelles » les éléments de la géographie physique d'une ville ou d'un ensemble métropolitain, comme le fait qu'il s'agit d'une île, par exemple, sa topologie (le terrain est-il plat ou accidenté, par exemple), son climat (la présence de neige et de froid en hiver) ou encore la proximité de montagnes ou d'autres limites à son expansion territoriale. Certaines de ces contraintes peuvent nuire au transport durable, comme le fait de devoir attendre un autobus en hiver par grand froid. Mais dans d'autres cas, les contraintes ont pu encourager un transport durable, comme le fait qu'une limite physique empêche l'étalement de la ville loin de son centre, qu'il y ait des montagnes toutes proches ou qu'il s'agisse d'une île. Dans le cas de Singapour, il est certain qu'une part appréciable de son succès vient du fait que cet État-île a une étendue limitée, qui empêche de voir la ville comme capable d'une extension géographique illimitée, limitée par la mer de tous les côtés. New York, du moins dans sa partie centrale, et aussi Hong Kong, sont dans une situation comparable. Parfois, on peut surmonter les barrières naturelles : à Montréal, on a contribué à l'étalement urbain par la construction d'infrastructures qui annulent la barrière naturelle qui viendrait de la présence de rivières et de fleuves. À Toronto, il y a bien une barrière qui vient du lac Ontario au sud de la ville, mais le terrain assez plat au nord favorise l'étalement. Le terrain accidenté de Lausanne l'empêche d'avoir autant de succès en transport actif (marche, vélo) que Genève ou Zurich. Les défis que représente un terrain accidenté jouent aussi contre le transport actif dans plusieurs villes d'Amérique latine, facteurs qui se conjuguent parfois à des facteurs sociologiques reliés à la sécurité et à la possibilité de violence.

En résumé, les contraintes naturelles jouent parfois contre le développement du transport urbain durable, mais elles peuvent tout aussi bien jouer en sa faveur, en particulier par les limites qu'elles posent quant à l'étalement urbain.

\section{Dans le cas de Singapour, il est certain qu'une part appréciable de son succès vient du fait que cet État-île a une étendue limitée, qui empêche de voir la ville comme capable d'une extension géographique illimitée, limitée par la mer de tous les côtés.}

\section{LES CONTRAINTES CRÉÉES PAR L'INTERVENTION HUMAINE}

Sous cette catégorie, on trouve un assez large éventail de facteurs. Nous verrons les facteurs considérés par les plus objectifs ou mesurables en premier.

Dans cet esprit, nous aborderons en premier lieu la forme urbaine de départ. En effet, toutes les villes, sauf de très rares exceptions (Brasilia serait un exemple d'exception), héritent d'une forme urbaine préexistante, laquelle est plus ou moins favorable au transport durable. Ainsi, la densité est considérée comme favorisant le transport durable, car elle favorise la rentabilité $\mathrm{du}$ transport en commun. Mais il faut aussi regarder comment cette densité est configurée. Si elle prend la forme de «corridors» ou de « couloirs », elle est d'autant plus favorable au transport en commun, et celui-ci peut être planifié pour épouser en quelque sorte la forme déjà existante, favorable à son développement. Le paysage métropolitain est-il par ailleurs, pour ses parties moins denses, déjà largement configuré en forme d'étalement urbain, ce qui est le cas de plus en plus de villes dans le monde, et constitue le principal challenge pour atteindre un transport urbain durable ${ }^{1}$. A-t-on assisté à du redéveloppement du centre urbain, plus coûteux, mais plus favorable au transport durable que le simple développement ${ }^{2}$, en redonnant vie aux quartiers centraux? Voilà autant de données de départ qui confrontent les décideurs publics dans leur effort de rationaliser la mobilité urbaine. Bien sûr, dans le passé, ces formes urbaines n'étaient pas des contraintes, mais des décisions plus ou moins conscientes, mais pour les décideurs d'aujourd'hui, qui en héritent, elles se présentent comme des contraintes avec lesquelles il « faut faire avec ». 
Le deuxième élément des contraintes créées par l'homme se trouve dans les contraintes de nature économique. Ces contraintes comportent plusieurs dimensions. Comme nous le faisons pour tous les facteurs, nous commençons par les facteurs sur lesquels les décideurs ont moins de prise. Nous mentionnerons au départ, donc, le type d'insertion de la ville dans l'économie mondiale. C'est une donnée qui n'est pas réellement nouvelle, mais on a pris conscience de son importance relativement récemment. Ainsi, on voit maintenant la ville comme participant directement à l'économie mondiale, au-delà de sa dynamique nationale. Dans cet esprit, on se demande si la ville est située dans une trajectoire de croissance, est-elle le siège de secteurs économiques en demande ou en progression? Offre-t-elle une vie culturelle et sociale intéressante qui peut attirer les élites économiques avec leurs activités économiques à la clé?

Dans le même esprit, mais dans un registre un peu différent, comment les élites économiques sont-elles intégrées dans l'administration de la ville? S'agit-il d'une administration de type corporatiste, où les élites économiques jouent un rôle essentiel, ce qui peut bien sûr constituer un avantage, mais aussi une contrainte?

Du point de vue économique, toujours, on peut se demander si la ville dans son ensemble est en situation de croissance ou de décroissance, et il faut aussi souvent se demander si cette croissance se déploie en son centre ou dans ses composantes périphériques. Bien sûr, dans la mesure où les projets de transport durable se portent sur des équipements coûteux, comme le métro ou le tramway, la base fiscale d'une ville est essentielle, à moins, bien sûr, de compter sur des contributions financières de niveaux de gouvernements séniors. Dans un environnement fiscal plus modeste, le développement du bus, parfois sous sa forme de BRT (bus rapid transit), demeure le seul choix possible en matière de transport public de masse.

Par ailleurs, et paradoxalement, un système de transport public peut prospérer justement parce que la ville contient des populations de niveau économique modeste qui n'ont d'autre choix que d'utiliser le transport de masse, donc un transport en principe plus écologique. Nous pensons ici à une ville comme Sao Paulo, assez prospère pour posséder un métro, mais contenant une partie importante de la population qui n'est pas assez riche, pour l'instant, pour se payer une automobile. Donc, du point de vue du transport durable, la prospérité peut avoir toutes sortes de conséquences. Une faible prospérité empêche de construire des équipements de qualité qui feront réellement compétition à l'automobile, ce que l'on réussit à faire par ailleurs dans plusieurs villes européennes, notamment dans plusieurs villes suisses. Par contre, cette même situation de faible prospérité garde en quelque sorte captive du transport en commun une clientèle trop peu fortunée pour se procurer une automobile.

La prospérité fiscale de la ville, et aussi sa prospérité générale, conditionnent aussi le type de développement et de «redéveloppement» possibles. Dans un environnement moins prospère, on développe la ville en poussant ses limites toujours plus loin, là où les terrains coûtent le moins cher, alors que dans un environnement plus prospère, on peut plus facilement redévelopper, un procédé plus coûteux, c'est-à-dire densifier des zones plus centrales, rendant par le fait même la ville plus favorable au transport en commun, tout en limitant sa dispersion.

Notre prochain élément des contraintes créées par l'intervention humaine se penche sur les questions sociologiques ou socio-économiques. Nous avons choisi de nous pencher ici sur des facteurs qui, tout en étant contraignants, sont néanmoins partiellement malléables, du moins théoriquement. Par contre, nous avons choisi de ne pas traiter de tendances lourdes sur le plan mondial, sur lesquelles on a peu de prise au plan local. Par exemple, une des tendances lourdes de notre époque, l'individualisation de la consommation, avec les droits à la mobilité vus comme une liberté fondamentale à la clé, ne sera pas traitée ici, car dans le cas d'une ville en particulier, on ne peut y changer grand-chose. Tournons nous donc vers des facteurs qui, quoique contraignants, peuvent dans une certaine mesure être travaillés par des autorités publiques locales. 
$\mathrm{Au}$ premier chef, au-delà des statistiques de revenus par habitant d'une ville ou d'un milieu métropolitain, nous devons considérer sa distribution. Dans un environnement où les écarts de revenus sont importants ou très importants, le transport en commun fait face à un challenge additionnel. Dans un environnement de fracture économique importante, comme on en trouve dans certains pays en développement, mais aussi aux États-Unis, les gens aisés ne sont pas portés à utiliser le transport en commun, où ils viennent en contact assez intime avec des gens que tout sépare. Cela peut affecter le tracé des lignes de transport en commun, car on peut faire le tracé d'une ligne qui passe essentiellement par des quartiers aisés, autant sur le plan du domicile que sur le plan des quartiers des affaires et du travail.

Dans le même esprit de facteurs sociologiques, il faut aussi se demander s'il y a d'autres éléments qui empêcheraient les gens d'être ensemble dans un même véhicule public. Il y a plusieurs cas qui correspondent à cette situation, des cas qui sont probablement sous-étudiés. Il peut y avoir, par exemple, des tensions ethniques ou religieuses entre groupes opposés. Nous n'avons qu'à penser à plusieurs villes du Moyen-Orient. Sans compter les cas où il n'est pas habituel, pour ne pas dire interdit, pour des hommes et des femmes qui ne se connaissent pas d'emprunter le même véhicule, dans une situation de proximité forcée.

\section{LES DYNAMIQUES INSTITUTIONNELLES}

\section{Les difficultés de plusieurs villes nord- américaines sur le plan du transport durable s'expliquent par la place limitée qu'on est prêt à donner aux autorités municipales et métropolitaines en termes de planification du territoire.}

Dans le cas des dynamiques institutionnelles, nous procéderons, ici aussi, en partant des éléments sur lesquels nous avons moins de prise, et nous verrons dans un deuxième temps ceux sur lesquelles une action volontariste est plus réalisable.
Ceci nous amène à dire un mot sur les «gated communities », ces zones urbaines résidentielles qui choisissent de se séparer du reste de la ville par des murs et des mécanismes divers. Présentes surtout, actuellement, aux États-Unis, elles pourraient se développer davantage ailleurs, à la faveur de l'élargissement de l'écart de revenus dans un environnement de compétition mondiale exacerbée. Ce développement est tout à fait contraire au paradigme du transport urbain durable, et en constitue même son antithèse, tant il est évident que l'on imagine difficilement ses résidents se rendre à leur «gated community » en transport collectif. C'est bien sûr en automobile que l'on entre et on sort de ces « communities » qui n'ont de communauté que la fiction du nom.

En ne tenant pas compte de ces éléments sociopolitiques et sociologiques, on risque d'ignorer des facteurs importants de succès ou d'insuccès du transport en commun. En effet, à quoi servirait-il de construire un système sophistiqué de transport en commun, métro ou rail léger, si son tracé ou sa simple opération viole des interdits ou ignore des conflits sociaux ou ethniques très vivaces? Il faut donc faire appel à des équipes interdisciplinaires, qui sont prêtes à mettre l'effort et le temps pour comprendre les dynamiques urbaines du milieu où on opère, capables de prendre en considération le non-dit et le sous-entendu, avant de construire de coûteuses installations. Il ne faut pas seulement confier toutes les tâches à des ingénieurs.

Dans la catégorie des éléments plus difficiles à modifier, on peut mentionner les modes de gouvernance préexistants, profondément ancrés dans la culture nationale des pays où les villes se trouvent. En particulier, certains éléments de la culture nationale pèsent sur la possibilité pour un gouvernement d'intervenir dans la société de façon volontariste et proactive. En effet, il faut tenir compte du fait que toute intervention en transport urbain exige une intervention publique forte, d'une façon ou d'une autre. Les difficultés de plusieurs villes nord-américaines sur le plan $\mathrm{du}$ transport durable s'expliquent par la place limitée qu'on est prêt à donner aux autorités municipales et métropolitaines en termes de 
planification du territoire, où les spécialistes de ce domaine sont parfois considérés comme des suspects, des amoureux inconscients de l'intervention étatique. Pour mieux apprécier cette dimension, il faut consulter certaines données de la culture nationale plus large où baigne la ville, comme le World values survey ${ }^{3}$ qui nous donne des informations statistiques sur ce que les habitants d'un pays dans son ensemble considèrent comme légitime en termes d'intervention publique par exemple ou de rapports entre les secteurs privés et publics. Ici, la notion de «path dependency » est cruciale pour comprendre les opportunités, mais aussi les résistances possibles, devant une intervention publique proactive. Les débats sur la privatisation des services publics se situent dans ces éléments de gouvernance, même si, en pratique, ce sont parfois des questions secondaires dans un réel plan de transport urbain durable, puisque le secteur privé, dans une telle entreprise, sera toujours un peu secondaire ou subsidiaire. Nous pensons qu'il y a plusieurs façons de réussir un plan de transport durable, mais aucun ne se fie au secteur privé pour piloter l'ensemble du projet ${ }^{4}$. Ceci étant dit, plusieurs formules sont possibles et il y a plusieurs façons de réussir. Nous en savons assez déjà pour formuler quelques patterns de succès.

Un premier modèle de succès implique un maire très proactif, souvent charismatique, qui initie un projet radical de transport durable et le supervise du début jusqu'à la fin, avec l'aide d'un groupe restreint de technocrates et de spécialistes triés sur le volet.

Ainsi, un premier modèle de succès implique un maire très proactif, souvent charismatique, qui initie un projet radical de transport durable et le supervise du début jusqu'à la fin, avec l'aide d'un groupe restreint de technocrates et de spécialistes triés sur le volet. Ce dernier modèle est le modèle le plus simple. On en trouve des exemples en Amérique latine, en particulier ${ }^{5}$. Un deuxième modèle, que l'on trouve cette fois souvent dans certaines villes asiatiques, peut être appelé le modèle de planification intégrale. Il s'étend sur plusieurs années, en fait plusieurs décennies, et dépasse le charisme d'un seul individu pour son ancrage. Il est fortement basé sur une planification concomitante et même préalable du territoire, au point où le transport et la planification territoriale sont vus comme une seule et même politique publique. Cette planification ne se réalise pas d'une façon radicale, mais dans la durée. Il suppose une forte légitimité des autorités publiques. Un troisième modèle, que l'on trouve souvent en Europe, est celui que l'on appelle « Consultatif-dirigiste ». Il s'y trouve, bien sûr, une forte intervention publique, comme dans tous les cas de succès de transport durable, mais cette intervention publique, de type dirigiste-élitiste, est précédée de consultations publiques. Dans ce modèle, il y a une sorte d'équilibre entre les élites politicoadministratives, d'une part, et les dynamiques citoyennes, d'autre part, et il constitue autant un modèle «top down» que «bottom up ». Notre dernier modèle de succès est celui que nous appellerons le modèle "Consultatif-fragmentéinhibé ». C'est un modèle de succès qui réussit malgré ses faiblesses structurelles, malgré que la planification publique n'y soit pas appréciée et malgré une certaine fragmentation des autorités métropolitaines, qui rend la coordination de l'ensemble difficile. C'est un modèle qui réussit en dépit des contradictions et des difficultés qu'il rencontre, en dépit de lui-même d'une certaine façon. On trouve des exemples de ce modèle surtout en Amérique du Nord, dans des villes comme Boston, Chicago ou Seattle, qui réussissent à atteindre des parts modales en transport collectif très respectables selon n'importe quel critère international ${ }^{6}$.

Un élément sur lequel les autorités publiques ont plus de prise concerne l'intégration institutionnelle des autorités métropolitaines. Jusqu'ici, nous avons surtout référé aux villes, pour des raisons de commodité, mais il est certain que ce dont il est question, ce sont surtout des grands ensembles métropolitains, car la ville s'est étendue depuis maintenant plusieurs décennies. Dans ce contexte, nous pouvons affirmer qu'il n'y a pas de succès en transport urbain durable sans autorités métropolitaines fortes ${ }^{7}$, que cette autorité se limite au transport ou englobe aussi la 
planification territoriale, le deuxième cas étant plus souhaitable que le premier. En tant que facteur de succès, on aura compris pourquoi on le place après les patterns de gouvernance préexistants, car il nous semble nettement plus malléable, et presque toutes les grandes zones

\section{LE CHOIX DES POLITIQUES PUBLIQUES}

Avec cette catégorie des choix des politiques publiques, on entre dans des catégories de facteurs de plus en plus malléables. En effet, les autorités publiques peuvent plus facilement travailler au plan des politiques publiques qu'au niveau des contraintes culturelles dont elles héritent. Après tout, c'est pour réaliser des politiques publiques qu'on élit des gouvernements. Mais des objectifs plus malléables ne signifient pas que la partie soit gagnée d'avance. $\mathrm{Au}$ préalable, il aura fallu s'entendre sur des objectifs stratégiques. Ces objectifs stratégiques n'auront pas toujours pour nom «transport urbain durable ». Quand ils auront pour objectif officiel une diminution de la congestion, leurs implications positives sur le transport durable seront moins évidentes, et il faudra donc regarder les détails de la stratégie. Après tout, on peut penser que l'on réduit la congestion par l'ajout de voies et de routes pour le transport automobile, ce qui ne va guère dans le sens du transport durable.

Des politiques plus favorables au transport urbain durable pourront porter différentes étiquettes: Transit Oriented Development, Smart Growth, politique de densification, de redéveloppement, ou encore de créations ou de consolidations de corridors ou de couloirs urbains. Ce qui est important, du point de vue du transport urbain durable, c'est que l'on ait comme objectif ultime d'encourager un transfert des parts modales, du transport motorisé individuel vers d'autres catégories, que ce soit le transport en commun ou des formes de transport actif, plus spécifiquement, la marche et le vélo.

Dans la mise à l'agenda et les décisions publiques qui prépareront ces dernières politiques, il aura été préférable, surtout dans les urbaines se sont dotées de ces entités métropolitaines, territoriales ou régionales plus larges, sans toutefois toujours leur donner les pouvoirs et les ressources pour réaliser leurs missions, dont celles du transport, qui nous intéressent ici.

villes qui ont déjà une tradition de participation publique, d'en arriver à des décisions qui auront reçu une certaine approbation des intéressés, du public, bien sûr, mais aussi, et peut-être surtout, des parties affectées directement par les projets. Ainsi, une des recettes du succès sera d'avoir, à la même table, des groupes aux intérêts opposés, des riverains, des groupes écologistes et des associations d'automobilistes et de transport de marchandises, par exemple.

Même si l'objectif peut paraître simple, voire simpliste, il est prouvé, chiffres à l'appui, que de «sortir les gens de leur voiture » pour les amener vers d'autres formes de mobilité constitue le point central de toute politique publique de transport urbain durable. Rappelons que $90 \%$ de la différence en terme de production de gaz à effet de serre d'une ville à l'autre s'explique par la différence dans l'utilisation de l'automobile ${ }^{a}$, à tel point que George Monbiot a estimé que si, par un tour de magie, on transférait tous les citoyens d'une ville complètement dominée par l'automobile vers le transport en commun, on réduirait de $90 \%$ la production de gaz à effet de serre due au transport dans cette ville ${ }^{\mathrm{b}}$. Il est rare en politiques publiques d'avoir un critère si évident et si objectif, qui montre la voie à suivre de façon aussi fiable. Mais si l'objectif est clair, les moyens pour y parvenir n'en sont pas par contre évidents. C'est l'objet de notre dernière section.

$\mathrm{Si}$, par un tour de magie, on transférait tous les citoyens d'une ville complètement dominée par l'automobile vers le transport en commun, on réduirait de $90 \%$ la production de gaz à effet de serre due au transport dans cette ville. 


\section{LE CHOIX DES INSTRUMENTS DE POLITIQUES PUBLIQUES}

Cette catégorie de facteurs de succès représente celle où les autorités publiques ont le plus de possibilités d'interventions concrètes. Autrefois vu comme une simple question administrative de détail, le choix des instruments de politiques publiques est devenu dans les quinze dernières années une question de tout premier ordre en analyse de politiques publiques.

On peut les définir comme l'ensemble des moyens à la disposition des gouvernements pour affecter les actions et comportements des acteurs sociaux dans un sens souhaité par ces autorités publiques. Les définitions varient quelque peu d'un auteur à l'autre ${ }^{c}$, mais on s'entend généralement sur une liste, plus ou moins longue, qui comprendrait par exemple la réglementation, les incitatifs économiques, les accords volontaires ou la publicité gouvernementale.

Dans des politiques publiques complexes, comme l'atteinte d'un transport urbain durable, plusieurs types d'instruments de politiques publiques doivent être mis en branle, dans des séquences qui peuvent varier d'une ville à l'autre. Nos recherches nous suggèrent jusqu'ici certains patterns communs, mais aussi des différences importantes.

\section{Dans des politiques publiques complexes, comme l'atteinte d'un transport urbain durable, plusieurs types d'instruments de politiques publiques doivent être mis en branle, dans des séquences qui peuvent varier d'une ville à l'autre.}

Presque toutes les villes commencent par des interventions minimales en matière de transport, en construisant ou faisant construire des voies publiques de base pour les usages individuels.

Il s'agit donc, le plus souvent, d'une période d'intervention minimale, parfois complétée par quelques interventions de transport public. Le plus souvent, suit une période d'augmentation importante de l'utilisation de l'automobile, à laquelle les autorités, en mode réactif, tentent de s'adapter du mieux qu'elles le peuvent. Même des villes qui sont parvenues, plus tard, à des succès spectaculaires en matière de transport urbain durable, sont passées par cette période de non-intervention ou d'intervention modeste. Suit, généralement, une phase informative, où les autorités publiques, par différents moyens d'information, mesurent l'ampleur et les défis que représente le choix d'une politique de transport urbain durable. C'est ce qui suit cette phase informative qui est important pour nous ici. Car, dans les villes à succès en transport durable, les instruments de politiques publiques pour mettre en œuvre leurs objectifs varient substantiellement, à la fois dans leur importance relative et dans leur séquence précise. Par ailleurs, on peut dire que, quelle que soit leur séquence ou leur importance relative, ce sont trois types d'instruments assez définis qui seront presque toujours utilisés, soit des instruments proactifs, incitatifs et consultatifs.

Toutes les villes utiliseront des instruments proactifs, comme la planification territoriale, le zonage, la construction d'infrastructures ou les réglementations sur le transport dans le centreville. Pour ce qui est des deux autres catégories, il y aura plus de variation. En effet, certaines villes à succès feront peu appel à la consultation publique, notamment en Amérique latine, d'autres beaucoup, comme Seattle par exemple ${ }^{8}$. Certaines villes à succès utiliseront intensément les incitatifs économiques, par des écotaxes substantielles ou des échanges et achats de permis, comme Singapour, d'autres peu ou même pas du tout. Il y a donc des chemins différents pour atteindre les mêmes buts.

Pour les villes qui veulent apprendre des villes qui connaissent du succès en la matière, il y a donc plusieurs modèles disponibles, et non pas un seul. Elles peuvent donc choisir le modèle qui est plus cohérent avec leur propre tradition de gouvernance, augmentant par le fait même leur chance de succès, en diminuant les résistances institutionnelles, toujours possibles. 


\section{CONCLUSION}

\section{Les facteurs de succès de la diminution des gaz à effet de serre dépendent donc en}

bonne partie du succès de nos institutions à modifier nos formes de mobilité. C'est une tâche énorme, mais il est bon de savoir que certaines villes ont su relever ce défi, par des chemins différents

Nous avons voulu, dans ce texte, faire un inventaire exhaustif des contraintes, mais aussi des facteurs de succès, qui se présentent pour toutes les villes du monde dans leur recherche d'un transport urbain durable. Notre prétention est à l'effet qu'aucun type de facteurs n'est absent, même si, à l'intérieur des catégories que nous proposons, tous les sous-types n'y sont pas nécessairement déclinés. Dans une étape ultérieure de notre recherche, nous pourrions modéliser ces facteurs, pour en déterminer par exemple des séquences précises ou leur poids relatif, pour prévoir des probabilités et des degrés de succès.

Notre but peut aussi être considéré comme scientifique, de par son intérêt pour les patterns de gouvernance par exemple, mais il se veut aussi pratique, par la spécification des différents instruments de politiques publiques, ainsi que leur séquence, pour atteindre le transport urbain durable.

Le transport urbain peut être un facteur qui contribue au succès de la diminution des gaz à effet de serre en transport, mais il peut aussi contribuer à son échec. Nous prenons pour acquis que les changements technologiques seront insuffisants ou arriveront trop tard pour changer la donne de façon notable. Les facteurs de succès de la diminution des gaz à effet de serre dépendent donc en bonne partie du succès de nos institutions à modifier nos formes de mobilité. C'est une tâche énorme, mais il est bon de savoir que certaines villes ont su relever ce défi, par des chemins différents, desquels nous pouvons nous inspirer. Notre recherche sur ce sujet est un «work in progress », mais nous avons voulu ici partager les conclusions provisoires auxquelles nous sommes parvenues.

\section{BIBLIOGRAPHIE ET NOTES}

${ }^{1}$ Mees, Paul (2010). Transport for Suburbia: Beyond the Automobile Age, Londres et Sterling, Virginia: Earthscan.

${ }^{2}$ Filion, Pierre (2010). "Intensification and Sprawl: Residential Density Trajectories in Canada's Largest Metropolitan Regions", Urban Geography, vol. 31: 541-569.

${ }^{3}$ World Values Survey Association (2009). Official Aggregate v. 2009091. World Values Survey 19812008. Disponible à: http://wwww.worldvaluessurvey.org. Aggregate file producers: ASED/JDS, Madrid.

${ }^{4}$ Mercier, Jean (2009). "Equity, Social Justice, and Sustainable Urban Transportation in the Twenty-First Century", Administrative Theory and Praxis, vol. 31(2): 145-163.

${ }^{5}$ Carrier, Mario, Jean Mercier, Fabio Duarte, Julien Domingue et Fanny T. Racicot (2011). « Instruments de gouvernance pour un transport durable dans les régions métropolitaines des Amériques : les cas de Seattle (États-Unis) et de Curitiba (Brésil) », dans LucNormand Tellier et Carlos Vainer (dir.), Métropoles des Amériques en mutation. Montréal : Presses de l’Université du Québec, pp. 209-233.

${ }^{6}$ Carrier, Mario et al., op.cit.

${ }^{7}$ Mees, Paul, op. cit.

${ }^{8}$ Carrier, Mario et al., op. cit.

Notes

${ }^{a}$ Cette observation a d'abord été formulée par Daniel Sperling dans ses comparaisons internationales des années 1990.

${ }^{\mathrm{b}}$ Il s'agit essentiellement de la même observation que celle faite par Daniel Sperling, mais exprimée un peu différemment. Elle vient de MONBIOT, George, (2006), Heat-How to Stop the Planet from Burning, Toronto, Doubleday/Random House, p.147.

${ }^{\mathrm{c}}$ Pour une définition, voir entre autres : Lester M. Salamon (2002). The Tools of Government: A guide to the new governance. New-York: Oxford University Press. 
2013

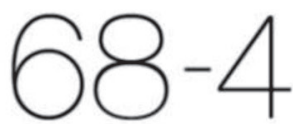

\section{REVUE TRIMESTRIELLE} RELATIONS INDUSTRIELLES

Revue trimestrielle bilingue publiée depuis 1945 par le Département des relations industrielles de l'Université Laval

\section{NUMÉRO THÉMATIQUE}

Le Réseau de recherche en santé et sécurité du travail du Québec : un bilan de dix ans

The Quebec Occupational Health and Safety Research Network: A Ten Year Update LISE DESMARAIS ET GABRIELLE LEGENDRE

ARTICLES

Le transfert de connaissances au RRSSTQ : bilan et perspectives

M. LORTIE, L. DESMARAIS, C. FAYE, E. LAROCHE ET I. FAURIE

Les conditions de travail au Québec : une analyse différenciée selon les groupes d'âge

E. LEDOUX, P.S. FOURNIER, D. CHAMPOUX, P. PRUD'HOMME, M. LABERGE, C. AUROUSSEAU, S. OUELLET ET C. CHATIGNY

From Serious Shortage of Researchers to OHS Law Changes and Growing Industrial Needs: A Dynamic and Quiet Force

S. NADEAU, J. FATISSON, A. BADRI AND B. EMAMI-MEHRGANI

Advancement in Quebec Research on the Prevention of Risks Related to Occupational Exposure to Nanomaterials

P. DOLEZ, Y. CLOUTIER, A. NOEL, L. TABET, D. GAUTRIN, M. DEBIA, G. L'ESPERANCE, S. HALLE, A. BAHLOUL. AND L. VINCHES

Quebec Research on Work-related Musculoskeletal Disorders:

Deeper Understanding for Better Prevention

J. N. COTE, S. NGOMO, S. STOCK, K. MESSING, N. VEZINA, D. ANTLE, A. DELUSLE, M. BELLEMARE, M. LABERGE AND M. ST-VINCENT

Santé organisationnelle : où en sommes-nous et vers où allons-nous au Québec?

V. DAGENAIS-DESMARAIS, M.E. DUFOUR, F. ST-HILAIRE ET R. HEBERT

Bilan relatif aux dispositions de la LSST : vers une application intégrale? G. BARIL-GINGRAS, M. VEZINA ET K. LIPPEL

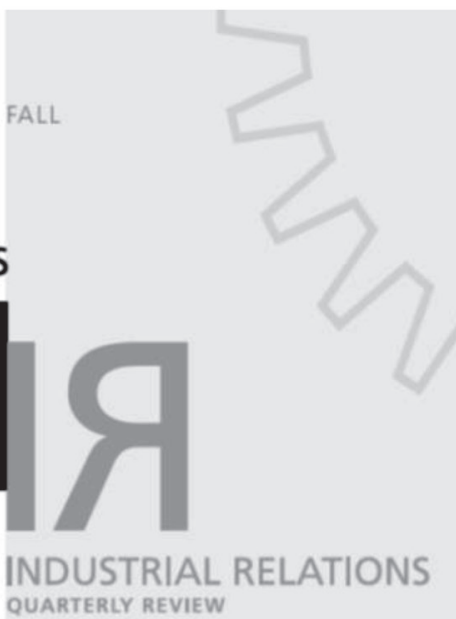

A bilingual quarterly published since 1945 by the Industrial Relations Department, Université Laval

\section{RI/IR EN LIGNE}

RI/R est disponible en ligne sur le site Erudit :

unw.erudit.org/revue/ri

Pour abonnement à la version numérique, contacter Érudit.

Pour consulter les sommaires des numéros publiés, les résumés d'articles ou pour vous abonner à la version papier, visitez notre site Internet :

www.riir.ulaval.ca

\section{RI/IR ONLINE}

RI/R is available on line on Erudit website at: unw.erudit.org/revue/ri

To subscribe to the online version, please contact Erudit.

Visit our website for contents listings, abstracts, or to subscribe to the print edition:

wuw.riir.ulaval.ca

\section{RELATIONS INDUSTRIELLES} INDUSTRIAL RELATIONS

Pavillon J.-A.-DeSève 1025, avenue des Sciences-Humaines Bureau 3129, Université Laval Québec (Québec) Canada G1V OA6 TELLEPHONE : (418) 656-2468 COURRIEL / E-MAIL : relat.ind@rit.ulaval.ca

www.riir.ulaval.ca 